\title{
Programa de Cinemática Directa con fines Educativos
}

\section{Direct Kinematic Program with Focus on Education}

\author{
Richard Parrales, Jacob Cuesta y Carlos Flores-Vázquez* \\ Unidad Académica de Ingeniería Industria y Construcción, Ingeniería Eléctrica \\ Universidad Católica de Cuenca, Cuenca, Ecuador \\ *cfloresv@ucacue.edu.ec
}

\begin{abstract}
Resumen
El propósito de este artículo es simplificar la manera de aprender y aplicar los pasos Denavit-Hartenberg (D-H) para el cálculo de la cinemática directa de un robot industrial. La aplicación descrita tiene la ventaja de simplificar significativamente el proceso del cálculo matemático que se realiza para encontrar la cinemática directa de dicho robot, al ingresar los parámetros D-H $(\theta, \mathrm{d}$, a y $\alpha)$ en el programa, este tiene la capacidad de mostrar los resultados previos a la matriz T (matriz de transformación homogénea). El manejo y utilización de la aplicación es intuitivo para las personas que tienen algún conocimiento en la ingeniería.
\end{abstract}

Palabras clave: Robótica, Cinemática Directa, Matriz de Transformación Homogenea, Cálculo Matricial..

\begin{abstract}
The purpose of this article is to simplify the way of learning and applying the Denavit - Hartenberg (D-H) steps for calculating the Direct Kinematics of an industrial robot. The application described has the advantage of significantly simplify the mathematic calculation process that is performed in order to find the direct kinematic of that robot. When entering the $D$-H parameters $(\theta, d$, a $y \alpha)$ into the program, this has the capacity to show previous results to the $T$ matrix (Homogeneous Transformation Matrix). The management and use of the application is intuitive for the people who have some knowledge in engineering.
\end{abstract}

Key words: Robotic, Direct Kinematics, Homogeneous Transformation Matrix, Matrix Calculation.

\section{INTRODUCCIÓN}

Para los estudiantes y profesionales que tienen conocimientos de Ingeniera conocen el inconveniente que se tiene al resolver las matrices de la cinemática directa de manera manual, por lo cual en la actualidad se han desarrollado varios programas de diferentes aspectos y modelos con el fin de minimizar el tiempo que se emplea para la resolución de cinemática directa.

Varios programas se han enfocado específicamente en un solo tipo y modelo de robot, así como en un número fijo de grados de libertad (GDL) sin posibilidad de cambiarlos por el usuario, mientras que otros programas se han enfocado solo en el aspecto de cálculo y respuesta sin posibilidad de visualizar los pasos intermedios para el desarrollo de la cinemática directa [1] [2].

Para la elaboración de programas en cinemática directa de un robot tipo industrial en ingeniería, los autores le han puesto mayor preocupación al aspecto matemático y no al proceso didáctico para la elaboración de programas educativos.

Como se sabe, es necesario implementar un programa con aspectos teóricos ya que serán de ayuda para los estudiantes que se encuentran en el proceso de aprendizaje, así como los profesionales que necesitan recordar la cinemática directa. Por ello se debe implementar un programa que incluya la parte teórica de cálculo en la cinemática directa de un robot, donde se indiquen como aplicar el algoritmo D-H, así como las matrices de transformación homogénea en un robot industrial.

Tantaruna, Medrano y Luna exponen el aspecto teórico de la Cinemática Directa de un robot, así como el aspecto práctico donde elaboran una maqueta de un robot industrial. En aquel programa usan la teoría para crear la parte matemática de la cinemática directa de un robot por lo cual no indica en dicho programa los pasos a seguir de manera teórica para obtener la cinemática directa [3]. Entonces es necesario que en un programa educativo incluya los aspectos teóricos de la cinemática directa de un robot.

En ciertos artículos con fines educativos se han desarrollado modelos matemáticos de la cinemática directa con el fin de obtener los resultados de posición en cada articulación [4] [5]. Estos documentos se basaron en robots manipuladores reales para obtener la cinemática directa y de esa manera sirva como aporte educativo para las personas que se encuentran en proceso de aprendizaje en ingeniería. Estos cálculos matemáticos se desarrollaron utilizando el 
algoritmo de D-H para obtener las matrices de la cinemática directa.

Un aspecto que no contemplan estos artículos con fines educativos [4] [5] es que no poseen el desarrollado de un interfaz gráfica para el cálculo matemático de la cinemática directa para distintos robots y con GDL variables, sino que solo obtienen las soluciones matemáticas de robots específicos con GDL fijos, normalmente emplean código para que el usuario ingrese los datos.

El presente artículo se enfocará en la resolución de robots tipo industrial de diferentes modelos y hasta seis GDL, es decir, que en este programa se puede resolver robots desde uno a seis GDL. Además, el programa facilitara la resolución matemática y teórica de la cinemática directa para reducir el tiempo empleado por las personas que requieran soluciones en problemas de este tipo.

Programas similares se han realizado en diferentes universidades, pero el aquí presentado tiene como finalidad el realizar los cálculos matemáticos de la cinemática directa para poder observar numéricamente que sucede con el robot y no solo visualizar en pantalla el movimiento del mismo, como es el caso del programa presentado en el artículo de la referencia [6] .

En la parte teórica del programa se tomará en cuenta los 16 pasos del algoritmo de Denavit-Hartenberg [7] [8] [9] [10] [11] [12], como también la visualización de vídeos educativos que ayuden a reforzar los conocimientos del usuario.

Mientras que en la parte matemática se presentara la facilidad de resolver las matrices de transformación de la cinemática directa, con la ventaja de no recurrir a la resolución matemática de manera manual.

Un programa tiene similares características con este documento donde la interfaz gráfica visualiza el movimiento del robot sin mostrar los resultados de forma numérica. La diferencia en este programa es que se visualiza las matrices en su forma numérica con una interfaz en pantalla para saber qué pasa con dicha matriz, evidenciando cambios en la posición del robot.[13]. Dicho programa citado, ayudo a la comprensión de herramientas a utilizar en el programa presente.

Los sistemas que ayudan a la comprensión de robótica estudiantil son escasos y poco comprensibles para los estudiantes que están iniciando en esta materia, por ello se ha desarrollado varios algoritmos que permiten el estudio más didáctico del mismo [14], lo cual permitió plantear este proyecto como un complemento a la ayuda educativa y de intuitiva comprensión en la aplicación de cinemática directa y sus efectos matemáticos al momento de posicionar al robot en diferentes ángulos.

\section{MetodologíA}

El presente documento hará referencia a la especialidad de robótica con respecto al cálculo de la cinemática directa a través de un programa dirigido a estudiantes y profesionales de Ingeniería.

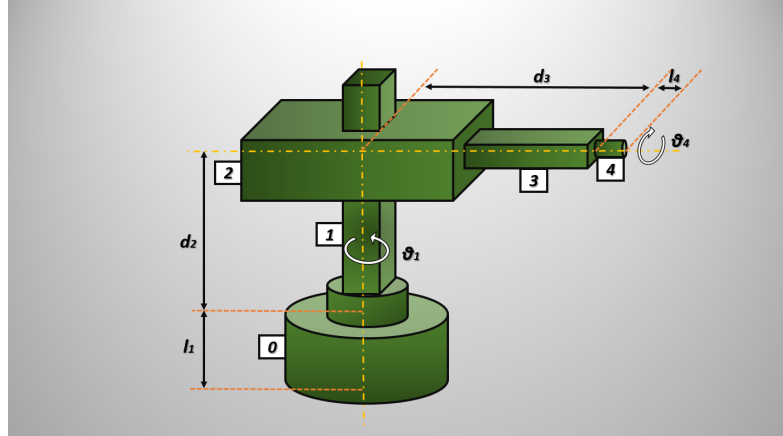

FIG. 1. Calculo de grados de libertad (GDL) de un robot cilíndrico.

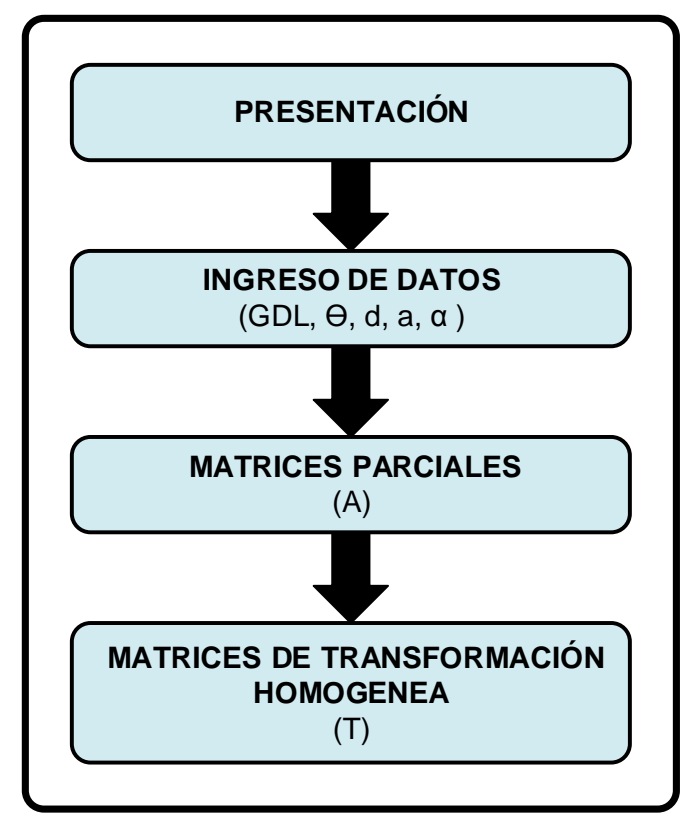

FIG. 2. Diagrama del programa numérico de cinemática directa.

Este programa posee un enfoque para contrarrestar el problema del tiempo que toma en resolver matemáticamente la cinemática directa de manera manual. Para aquello los robots que pueden resolverse mediante este programa son de tipo industrial con características de hasta seis GDL.

Los grados de libertad (GDL) se obtiene a través de enumerar cada articulación comenzando por 1 y terminando en " $n$ ". Por ejemplo un robot cilíndrico cuenta con cuatro articulaciones, es decir, con cuatro grados de libertad como se observa en la Fig. 1.

Para realizar el programa primero se debe identificar los aspectos necesarios que ayudaran entender y calcular la cinemática directa de un robot industrial, por lo cual en la Fig. 2 se representa en forma de diagrama el proceso de cálculo del programa.

Para la creación y entendimiento del programa, esta se 


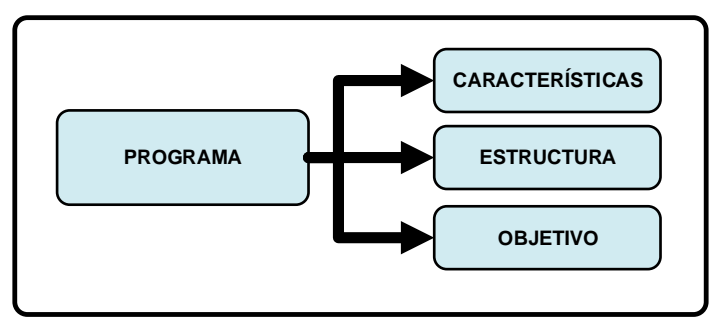

FIG. 3. División del programa de cinemática directa.

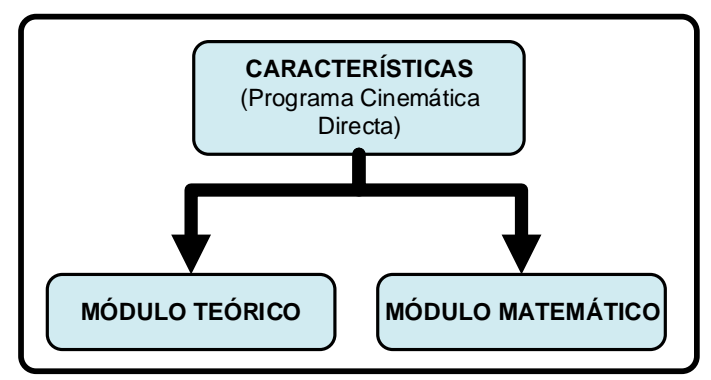

FIG. 4. Características del programa de cinemática directa.

dividió en tres partes importantes que son las características, la estructura y su objetivo como se observa en la Fig. 3.

\section{A. Características}

En las características del programa de la cinemática directa se consideró la elaboración de dos partes importantes que son el modulo teórico didáctico y el módulo matemático Fig. 4, esto es para que el usuario tenga facilidad de comprensión con la temática abordada en el programa y de esa manera no tenga complicaciones al momento de usarla.

En la parte del módulo teórico comprende la explicación de los pasos del algoritmo de $\mathrm{D}-\mathrm{H}$, mientras que en la parte del módulo matemático comprende la resolución de cinemática directa donde la persona interactúa con el programa para encontrar las matrices de transformación del robot industrial que pueden ser desde uno hasta seis GDL.

\section{B. Estructura}

Para la estructura del programa de la cinemática directa se considera la utilización de GUIDE MatLab una herramienta de desarrollo para crear el programa. La estructura del programa está orientada a la descripción del diseño como lo ilustra la Fig. 5.

Las características del programa se comprende de dos módulos, uno teórico y otro matemático, en la parte teórica como se muestra en la Fig. 6 su estructura comprende en la explicación de los 16 pasos del algoritmo de Denavit Hartenberg (D-H) [7], que son los siguientes:

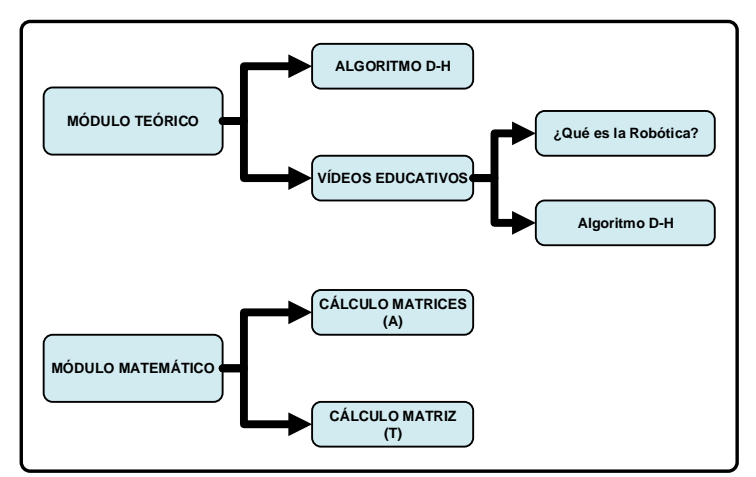

FIG. 5. Estructura del programa de cinemática directa.

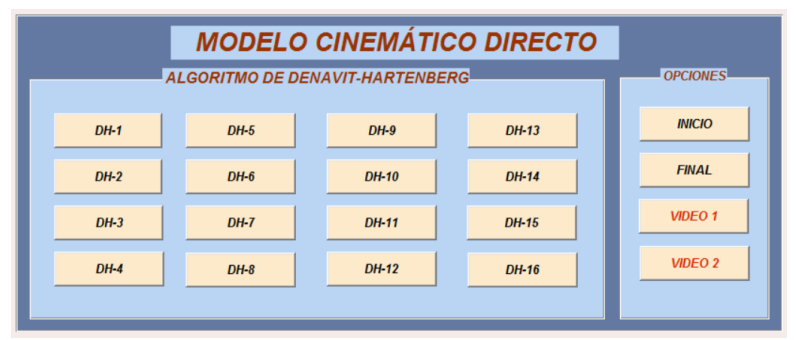

FIG. 6. Interfaz gráfica del módulo teórico del programa.

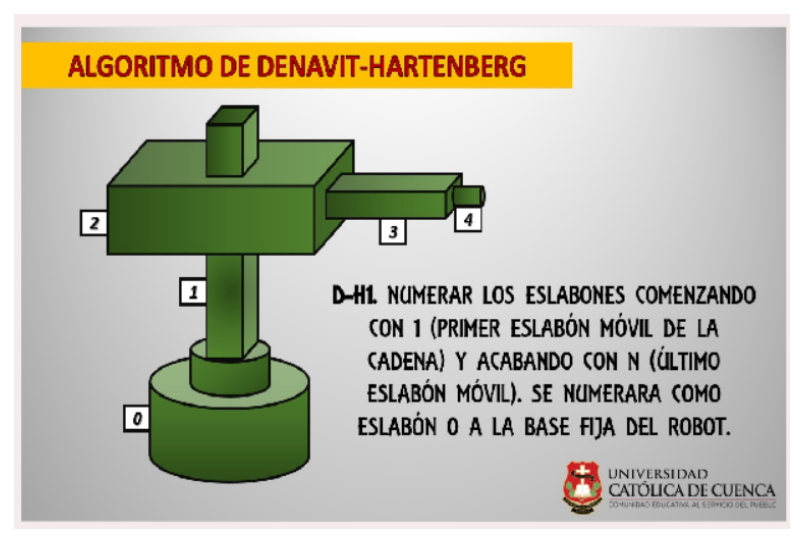

FIG. 7. Ejemplo de visualización en pantalla de un paso D-H.

- Paso 1 (D-H 1) al paso 9 (D-H 9): Identificación de la estructura del robot, así como situar el sistema de coordenadas.

- Paso 10 (D-H 10) al paso 13 (D-H 13): Obtener $\theta, \mathrm{d}, \mathrm{a}$ y $\alpha$ del robot industrial correspondiente.

- Paso 14 (D-H 14) al paso 16 (D-H 16): Obtener las matrices de transformación A y $\mathrm{T}$ del robot.

Para la visualización de los pasos del algoritmo de D-H se creó una interfaz gráfica para mostrar dichos pasos a través de imágenes como se observa en la Fig. 7.

Adicionalmente en la construcción del diseño didáctico se agregó dos vídeos educativos en el primero llamado 


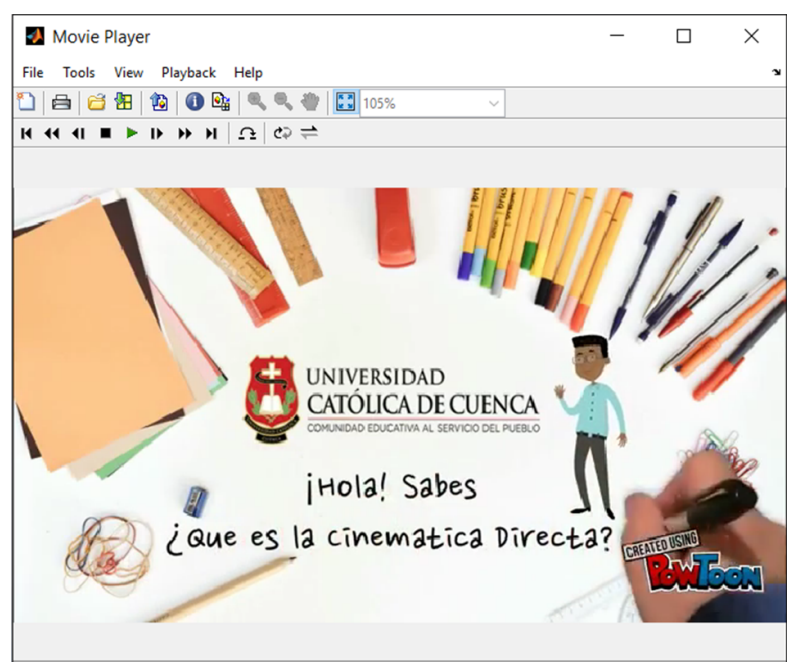

FIG. 8. Visualización del vídeo educativo.

“QQué es la robótica?” [15] y en el segundo “Algoritmo de Denavit-Hartenberg" [16] para que el usuario que interactúa con el programa tenga la facilidad de comprender lo que está realizando Fig. 8. Los vídeos se realizaron en PowToon.

En la parte matemática se construye todo lo que manipulara la persona para buscar soluciones de las matrices del robot industrial sin la necesidad de realizar sus soluciones matemáticas manualmente. Aquí la persona introduce los grados de libertar (GDL) que tiene el robot industrial, así como los valores obtenidos del paso 10 (D-H 10) al paso 13 (D-H 13) que son $\theta, \mathrm{d}$, a y $\alpha$.

Donde:

- $\theta$, es el ángulo que forman los ejes $X_{i-1}$ y $X_{i}$ medido en un plano perpendicular al eje $Z_{i-1}$.

- d, es la distancia a lo largo del eje $Z_{i-1}$ desde el origen del sistema de coordenadas $(i-1)$-ésimo hasta la intersección del eje $Z_{i-1}$ con el eje $X_{i}$.

- $\quad \alpha$, es el ángulo de separación del eje $Z_{i-1}$ y el eje $Z_{i}$. [8]

De esa manera se obtendrá los resultados de la matriz de transformación homogénea del robot industrial que se haya elegido, teniendo la visualización de esta matriz en la parte inferior derecha del programa con su respectiva imagen que identifica la solución obtenida.

La fórmula de la matriz $\mathrm{T}$ y las matrices A utilizadas en el programa educativo son las que se muestran en la Fig. 9.

\section{El Objetivo del Programa}

Minimizar el tiempo de proceso matemático en forma escrita por parte del usuario que está calculando la cinemática directa del robot industrial.

De esta manera el programa puede ser usado por parte de estudiantes y profesionales que tengan conocimientos en la especialidad de robótica y ramas afines de la ingeniería, concentrando su atención por completo en el proceso de resolución D-H.

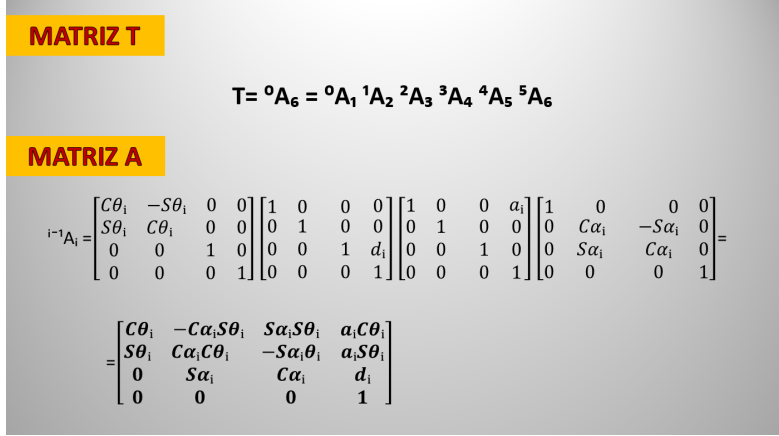

FIG. 9. Formula de la matriz T y A de la cinemática directa [8].

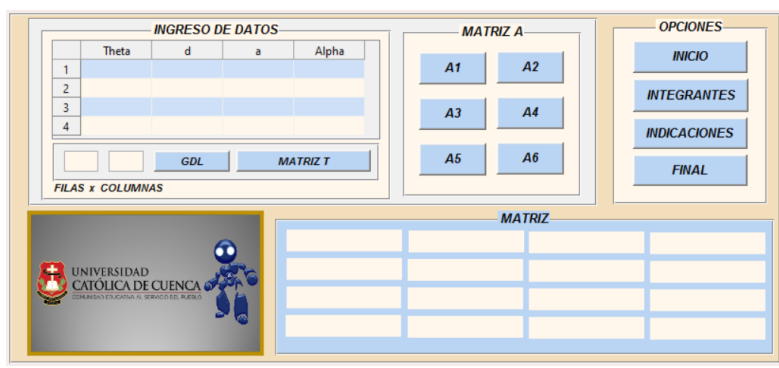

FIG. 10. Estructura de la parte matemática del programa.

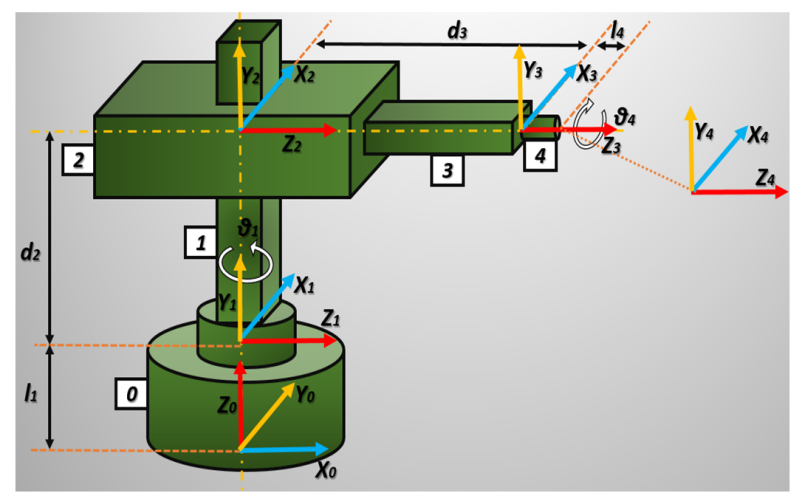

FIG. 11. Algoritmo D-H del robot cilíndrico.

\section{RESULTADOS}

Para la obtención de los resultados en este documento se tomaron los tres aspectos principales mencionados en la sección previa como son las características, la estructura y el objetivo del programa de la cinemática directa, el cual genero la interfaz gráfica ilustrada en la Fig. 10.

Entre los resultados se puede obtener el cálculo de la cinemática directa de un robot industrial de hasta seis GDL, así como la representación de los 16 pasos del algoritmo de Denavit-Hartenberg y la reproducción de dos vídeos educativos para reforzar los conocimientos de las personas que utilicen el programa. 


\begin{tabular}{|c|c|c|c|c|c|c|c|}
\hline & & & $-I N$ & RES & $D E$ & & \\
\hline & \multicolumn{2}{|c|}{ Theta } & & d & & a & Alpha \\
\hline 1 & \multicolumn{2}{|l|}{$q 1$} & 11 & & 0 & & 0 \\
\hline 2 & \multicolumn{2}{|c|}{$p i / 2$} & $d 2$ & & 0 & & $p i / 2$ \\
\hline 3 & \multicolumn{2}{|l|}{0} & d3 & & 0 & & 0 \\
\hline 4 & \multicolumn{2}{|l|}{$q 4$} & 14 & & 0 & & 0 \\
\hline & 4 & 4 & & $G D L$ & & & MATRIZ T \\
\hline & AS $x$ & -01 & & & & & \\
\hline
\end{tabular}

FIG. 12. Parámetros de D-H del robot cilíndrico

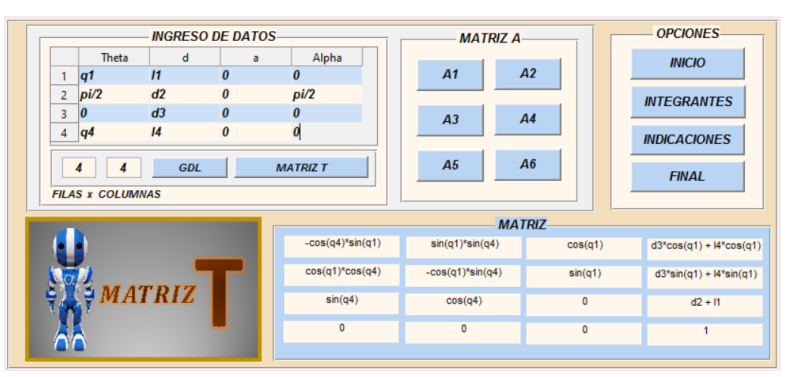

FIG. 13. Cálculo de la matriz T de un robot cilíndrico.

A. Resolución de la cinemática directa de un robot industrial de cuatro grados de libertad.

Se desarrollará la resolución de cinemática directa en un robot industrial cilíndrico de cuatro GDL para demostrar el funcionamiento del programa. La resolución del robot en el programa contempla los siguientes pasos:

1) Desarrollar el algoritmo de D-H Fig. 11.

2) Agregar el número de GDL del robot Fig. 12.

3) Ingresar los valores de $\theta$, d, a y $\alpha$ Fig. 12.

4) Calcular la matriz T Fig. 13.

5) Obtener las matrices A Fig. 14.

Es necesario realizar el algoritmo de D-H de un robot para identificar el número de GDL, así como el sistema de coordenadas correspondientes a cada eslabón.

Se recuerda que al momento de ingresar los datos de $\theta$, d, a y $\alpha$ al programa, estas deben ser en radianes y no en grados.

Para el cálculo de la matriz $\mathrm{T}$ de un robot, es necesario identificar el número de GDL del robot industrial para que se ingrese al programa de cinemática directa.

Al obtener el resultado de la matriz T, simultáneamente se obtiene cada matriz A del robot industrial.

El programa educativo de la cinemática directa provee de indicaciones de como calcular la matriz $\mathrm{T}$ y las matrices $\mathrm{A}$ de un robot industrial de hasta seis grados de libertad como lo indica la Fig. 15.

\section{CONCLUSIONES}

Los aspectos y pasos que se presentó para la creación $\mathrm{y}$ funcionamiento del programa han dado resultados favo-
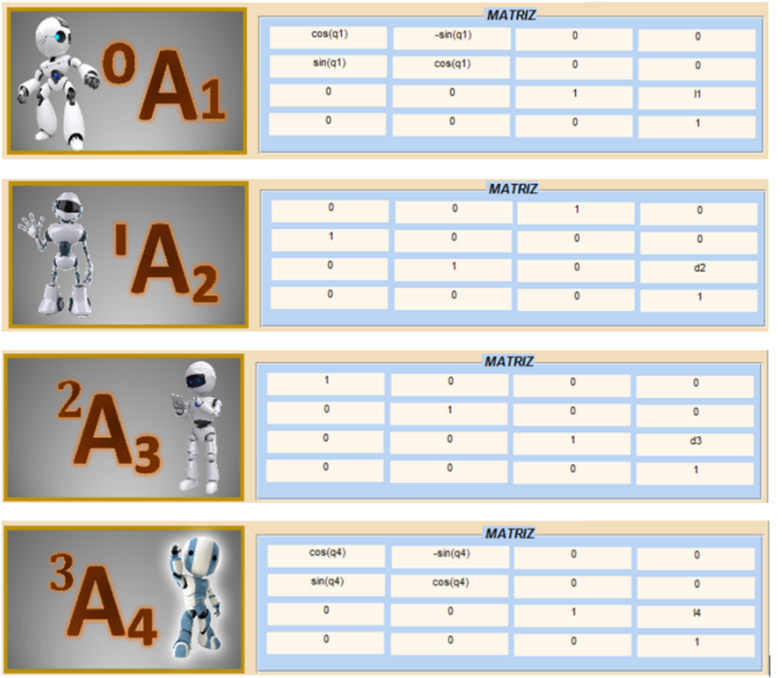

FIG. 14. Cálculo de la matriz A de un robot cilíndrico.

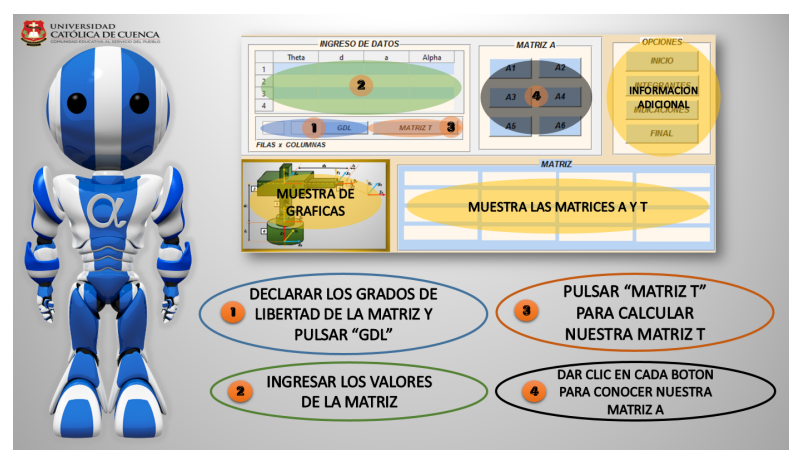

FIG. 15. Indicaciones de como calcular la matriz $\mathrm{T}$ y las matrices A del programa de la cinemática directa.

rables en la obtención de cinemática directa para robots industriales, el cual además de el proceso didáctico del algoritmo D-H ha permitido obtener la resolución matemática del posicionamiento de un robot industrial reduciendo el tiempo que el usuario empleaba.

Este programa brinda información educativa necesaria del algoritmo Denavit-Hartenberg como una herramienta de ayuda para el estudiante o persona profesional que requiera agilidad y ahorro de tiempo en el proceso en los cálculos de la cinemática directa de un robot tipo industrial y de diferentes modelos, además el programa es capaz de resolver robots que van desde uno hasta seis GDL.

\section{REFERENCIAS}

[1] M. T. Roberto, C. R. J. Salvador, H. R. Fernando, J. E. V. Soto, and J. C. P. Ortega, "Software de simulación de un robot manipulador marca mitsubishi rv-2aj," 4TO Congreso Nacional de Mecatrónica, 2005.

[2] J. Gallardo-Alvarado, J. M. Rico-Martínez, and H. Orozco-Mendoza, "Un algoritmo para resolver la cinemática directa de plataformas gough-stewart tipo 
6-3," Computación y Sistemas, vol. 8, no. 2, pp. 132 149, 2004.

[3] R. M. Tantaruna and M. C. Luna, "Modelo de enseñanza de robótica en instituciones de educación superior,"

[4] J. L. R. Arias and A. R. Fonseca, "Modelamiento matemático de la cinemática directa e inversa de un robot manipulador de tres grados de libertad," Ingeniería solidaria, vol. 8, no. 15, pp. 46-52, 2012.

[5] M. C. Páez, J. A. H. Salazar, A. J. M. Pérez, and C. A. P. Cortes, "Diseño, construcción e implementación de una plataforma robótica multifuncional con propósitos educativos-majad 1.0," Entre Ciencia e Ingeniería, no. 16, 2014.

[6] O. D. Morán and R. R. Monasterolo, "Enseñanzaaprendizaje en robótica: Construcción de simuladores como actividades de comprensión," Formación universitaria, vol. 2, no. 4, pp. 31-36, 2009.

[7] R. S. Hartenberg and J. Denavit, "A kinematic notation for lower pair mechanisms based on matrices," Journal of applied mechanics, vol. 77, no. 2, pp. 215-221, 1955.

[8] A. Barrientos, L. F. Peñin, C. Balaguer, and R. Aracil, Fundamentos de robótica. McGraw-Hill, Interamericana de España, 2007.
[9] B. Siciliano, L. Sciavicco, L. Villani, and G. Oriolo, Robotics: modelling, planning and control. Springer Science \& Business Media, 2010.

[10] P. Corke, Robotics, vision and control: fundamental algorithms in MATLAB, vol. 73. Springer, 2011.

[11] M. W. Spong, S. Hutchinson, and M. Vidyasagar, Robot modeling and control. John Wiley \& Sons, 2006.

[12] J. J. Craig, Introduction to robotics: mechanics and control, vol. 3. Pearson Prentice Hall Upper Saddle River, 2005.

[13] A. N. Chang Herrera, Diseño y Simulación de un Robot Articular con seis grados de Libertad utilizando el Toolbox Robotics de Matlab para fortalecer las clases teóricas realizando prácticas de laboratorio con el software presentado en este proyecto. $\mathrm{PhD}$ thesis, Quito: EPN, 2014., 2014.

[14] A. Majarena, J. Santolaria, D. Samper, and J. Aguilar, "Identificación de parámetros cinemáticos de una plataforma paralela pan-tilt basada en cinemática inversa y directa," Asociación Espanola de Ingenierı Mecánica, Ciudad Real, p. 186, 2010.

[15] R. Parrales and J. Cuesta, “¿qué es la robótica?.” https: //youtu.be/cM8Dl_6HEzY, 2017.

[16] R. Parrales and J. Cuesta, "Algoritmo de denavithartenberg." https://youtu.be/LmC5JnIaCms, 2017.

Recibido: 13 de enero de 2017

Aceptado: 15 de mayo de 2017

Richard Parrales Paladines: Es estudiante en la Unidad Académica de Ingeniería, Industria y Construcción perteneciente a la Universidad Católica de Cuenca, Ecuador. Correo electrónico: richafer19@hotmail.es

Jacob Cuesta Vazquez: Es estudiante en la Unidad Académica de Ingeniería, Industria y Construcción perteneciente a la Universidad Católica de Cuenca, Ecuador. Correo electrónico: jacob.ak47@outlook.com

Carlos Flores Vázquez: Es investigador en la Unidad Académica de Ingeniería, Industria y Construcción perteneciente a la Universidad Católica de Cuenca, Ecuador. 